\title{
Association between telomere length, frailty and death in older adults
}

\author{
Mariam El Assar • Javier Angulo • José A. Carnicero • Stefan Walter • \\ Francisco J. García-García • Fernando Rodríguez-Artalejo • \\ Leocadio Rodríguez-Mañas
}

Received: 10 July 2020 / Accepted: 20 October 2020 / Published online: 15 November 2020

(C) American Aging Association 2020

\begin{abstract}
Frailty is considered a clinical marker of functional ageing. Telomere length (TL) has been proposed as a biomarker of biological age but its role in human ageing is controversial. The main aim of the study was to evaluate the longitudinal association of TL with incident frailty and mortality in two cohorts of Spanish community-dwelling older adults. TL was determined at baseline in blood samples from older adults included in Toledo Study for Healthy Aging and ENRICA cohorts while frailty was determined by frailty phenotype (FP) at baseline and at follow-up (3.5 years). Deaths occurring during follow-up were also recorded. Associations of TL with frailty and mortality were analysed by logistic regression with progressive
\end{abstract}

Fernando Rodríguez-Artalejo and Leocadio Rodríguez-Mañas contributed equally to this work.

M. El Assar · J. A. Carnicero · S. Walter •

L. Rodríguez-Mañas

Fundación de Investigación Biomédica del Hospital Universitario de Getafe, Getafe, Spain

M. El Assar · J. Angulo · J. A. Carnicero •

L. Rodríguez-Mañas

Centro de Investigación Biomédica en Red de Fragilidad y Envejecimiento Saludable (CIBERFES), Instituto de Salud Carlos III, Madrid, Spain

\section{J. Angulo}

Servicio de Histología-Investigación, Unidad de Investigación

Traslacional en Cardiología (IRYCIS-UFV), Hospital

Universitario Ramón y Cajal, Madrid, Spain adjustment. Data were separately analysed in the two cohorts and in all subjects by performing a meta-analysis. TL was not different between frail and non-frail subjects. Longer telomeres were not associated with lower risk of prevalent frailty. Similarly, TL at baseline failed to predict incident frailty (OR: 1.04 [0.88-1.23]) or even the development of a new FP criterion (OR: 0.97 [0.90-1.05]) at follow-up. Lack of association was also observed when analysing the development of specific FP criteria. Finally, while frailty at baseline was significantly associated with higher risk of death at follow-up (OR: 4.08 [1.97-8.43], $p<0.001$ ), TL did not significantly change the mortality risk (OR: 1.05 [0.94-1.16]). Results show that TL does not predict incident frailty or

\section{F. J. García-García}

Hospital Virgen del Valle, Complejo Hospitalario de Toledo, Toledo, Spain

\section{F. Rodríguez-Artalejo}

Department of Preventive Medicine and Public Health,

Universidad Autónoma de Madrid/IdiPaz, CIBERESP, and

IMDEA Food Institute, Madrid, Spain

L. Rodríguez-Mañas

Servicio de Geriatría, Hospital Universitario de Getafe, Getafe, Spain

L. Rodríguez-Mañas $(\bowtie)$

Division of Geriatric Medicine, Hospital Universitario de Getafe,

Ctra de Toledo km 12,500, 28905 Getafe, Spain

e-mail: leocadio.rodriguez@salud.madrid.org 
mortality in older adults. This suggests that TL is not a reliable biomarker of functional age.

Keywords Ageing - Telomere - Functional decline . Frailty $\cdot$ Mortality $\cdot$ Biomarker

\section{Introduction}

Life expectancy has nearly doubled during the past century. As a result, population ageing is rapidly increasing worldwide: there were 703 million people aged 65 or over in 2019. The United Nations' report estimates that the global number of older persons will reach over 1.5 billion by 2050 . These demographic changes are more marked in Europe, where older persons will constitute more than one-third of the population at that time [1]. Recognizing that this phenomenon represents a major issue for the twenty-first century, one of the major challenges launched by the National Academy of Medicine of United States is directed at improving the health, the productivity and quality of life of older persons [2]. In this sense, disability and its preceding condition, frailty, are key determinants of healthy ageing.

One of the expressions of population ageing is the increase in the prevalence of frailty [3]. Frailty is a multi-systemic syndrome that reflects a state of decreased functional reserve and increased vulnerability to even minor external stressors, and is associated with increased risk of adverse health outcomes including hospitalization, morbidity, disability and death [4]. Frailty results from the interaction between the ageing process and some chronic diseases and conditions that are prevalent in older adults [5]. In fact, frailty has been suggested as a marker of biological ageing at clinical level [6].

Another widely used biomarker of biological age is telomere length (TL), although its role in human ageing is controversial [7]. Telomeres are involved in DNA replication and cellular ageing. Indeed, telomere attrition has been associated with increased risk of several age-related diseases [8] and death [9]. Telomeres are nucleoprotein structures found at the ends of eukaryotic chromosomes, and play a role in maintaining chromosomal integrity. They shorten during every cell division but, in addition to replication, other endogenous factors could result in telomere shortening including oxidative stress and inflammation, which are two underlying mechanisms of the ageing process and several agerelated diseases $[6,10]$.

However, because of the existence of conflicting results to date, there is no clear evidence of the relationship between TL and frailty. Some cross-sectional studies found that, in Caucasian older persons and in Asian older persons, TL was not associated with frailty assessed with the Fried criteria or the Frailty Index $[11,12]$. In contrast, other authors reported an inverse association between TL and frailty in older adults [13]. It should be noted that studies evaluating the association between basal TL and incident frailty are very scarce. In fact, only one population-based study reported the absence of association between TL and incident frailty after 4 years of follow-up in community-dwelling older Chinese individuals [11]. In fact, the need for longitudinal studies to elucidate the relationship between TL and frailty has been recently claimed [14].

Accordingly, in addition to evaluate the crosssectional relationship between TL and frailty, the main aim of this work was to ascertain if TL was longitudinally associated with incident frailty and with the risk of death in two cohorts of older community-dwelling individuals in Spain.

\section{Methods}

Study design and participants

Participants in this study included men and women aged $\geq 65$ years from two Spanish cohorts, namely, the Toledo Study of Healthy Aging (TSHA) [15, 16], and the Seniors-ENRICA cohort $[17,18]$. TSHA is a prospective cohort study initiated in 2006 with the aim of studying determinants and consequences of frailty in older adults residing in Toledo, Spain [15]. The ENRICA cohort was established in 2008-2010 with individuals selected through stratified random sampling of the population aged $\geq 60$ years in Spain [17].

Baseline information on socio-demographic data, health status, lifestyle, social support and morbidity was collected through face-to-face (TSHA) or phone (ENRICA) interviews. Also fasting blood samples were drawn and a physical examination, including physical and cognitive functioning, was performed at the participant's homes.

The TSHA study protocol was approved by the Clinical Research Ethics Committee of the Complejo 
Hospitalario de Toledo, and the ENRICA study by Clinical Research Ethics Committee of the 'La Paz' University Hospital in Madrid. All participants gave written informed consent.

Baseline data and blood samples for TL determination were collected in 2014-2015, and the follow-up expanded until the end of 2017 in both cohorts. For the present study, a nested case-control selection from both cohorts was performed at baseline. Cases were formed by all frail subjects in these cohorts, identified using the frailty phenotype (FP) (19), as described below. The controls were non-frail subjects randomly selected in a proportion of 4 non-frail to 1 frail. This resulted in a total of 439 individuals from the TSHA cohort of whom 84 (19.13\%) were frail and 355 non-frail. A total of 430 participants from the ENRICA cohort were included in this study, of whom 75 (17.44\%) were frail and 355 non-frail. TL was assessed in both cases and controls using the same techniques in the samples obtained at the time of the baseline examination of participants.

Frailty

Frailty was assessed in TSHA and ENRICA cohorts according to a slight modification of the definition of frailty phenotype proposed in the Cardiovascular Health Study (CHS) [19]. Frailty was defined as having at least three among the following five criteria: (a) unintentional weight loss of $\geq$ $4.5 \mathrm{~kg}$ in the preceding year; (b) exhaustion, based on a positive response to any of the following questions from the Centre for Epidemiologic Studies Depression Scale: 'I felt that anything I did was a big effort' or 'I felt that I could not keep on doing things' 'at least 3-4 days a week'; (c) weakness, defined as the cohort-specific lowest quintile of grip strength measured with a Jamar dynamometer in the dominant arm, and adjusted for sex and body mass index $\left(\mathrm{kg} / \mathrm{m}^{2}\right)$ [20] with the highest value in two consecutive measures selected for the analyses; (d) slow walking speed, defined as the cohort-specific lowest quintile in the 3-m walking speed test adjusted for sex and height [21]; and (e) low physical activity, defined as the lowest quintile in the Physical Activity Scale for the Elderly (PASE), adjusted for sex, in TSHA [22] and as walking $\leq 2.5 \mathrm{~h} /$ week in men and $\leq 2 \mathrm{~h} /$ week in women in ENRICA.
Disability

In both cohorts, limitations in basic activities of daily living (BADL) were assessed with the Katz Index [23]. The participants were considered disabled when they were dependent for at least one activity.

Follow-up period for incident frailty detection and for mortality identification

The mean follow-up time of participants of this study was 3.5 years. During this period, dates of death were obtained from the Spanish National Death Index (Ministry of Health and Social Service) for both TSHA and ENRICA participants. When necessary, deaths were confirmed by follow-up telephone interviews with relatives of the deceased. For assessment of incident frailty, data were available for 291 nonfrail subjects from TSHA cohort and 216 from ENRICA cohort.

DNA extraction

Genomic DNA was isolated from frozen blood samples as reported elsewhere [24] following a salting out procedure. Briefly, $500 \mu \mathrm{l}$ of blood were mixed with a hypotonic solution, washed twice with a detergent solution and finally precipitated in Ethanol $70 \%$ v/v. Samples were resuspended in Tris-EDTA buffer and kept at $4{ }^{\circ} \mathrm{C}$ until use. Sample absorbance at $260 \mathrm{~nm}$ was measured and concentration was calculated. Samples with more than $5 \mathrm{ng} / \mu \mathrm{l}$ and absorbance ratio 260/280 between 1.6 and 2.2 were considered optimal for TL calculation by qPCR.

Assessment of telomere length by real-time quantitative PCR method

TL was measured in DNA samples following an improved method based in that described by Cawthon [25] and O'Callaghan and Fenech [26]. By this method, telomere length is calculated as a ratio between telomere repeat copy number $(\mathrm{T})$ and a single-copy reference gene (36B4) (S). Then, we used cell lines with known telomere length to build a standard curve in which we can interpolate the results of our samples to obtain an absolute measure of the telomere length. TL of those cells were calculated by qPCR. The primer sequences (written $5^{\prime}$ to 
$\left.3^{\prime}\right)$ were as follows: telomere repeat-(TTAGGG)14; and 36B4-CAGCAAGTGGGAAGGTGTAA TCCGTCTCCACAGACAAGGCCAGGACTCGT TTGTACCCGTTGATGATAGAATGGG. Cycling conditions (for both telomere (T) and 36B4 (S) amplicons) were $10 \mathrm{~min}$ at $95{ }^{\circ} \mathrm{C}$, followed by 40 cycles of $95{ }^{\circ} \mathrm{C}$ for $15 \mathrm{~s}, 60^{\circ} \mathrm{C}$ for $1 \mathrm{~min}$. qPCR analysis was performed using the QuantStudio ${ }^{\mathrm{TM}} 5$ PCR System to estimate the Ct (threshold cycle), defined as the intersection between an amplification curve and a threshold line.

\section{Cell lines}

To perform the standard curve, lymphocyte cell lines, commercially available, were used: IM9 (ATCC-CCL159; batch number 59102678), CEM (ATCC-CCL-119; batch number 59102678) and REH (ATCC-CRL-8286; batch number 59429141) cell lines were purchased from American Type Culture Collection (ATCC; Manassas, VA, USA). C0106 (ECACC-91071212; batch number HB1489) cell line was obtained from ECACC. COJ cell line was established from a healthy donor. Cell lines were grown following manufacturer's conditions (RPMI 1640 growth media) supplemented with $10 \%$ (v/v) foetal bovine serum (FBS), $2 \mathrm{mM}$ glutamine, and penicillin/streptomycin $0.1 \mathrm{mg} / \mathrm{ml}$ and $1 \mathrm{mM}$ sodium pyruvate and $10 \mathrm{mM}$ HEPES for the REH cell line. Grown cultures were frozen in 90\% FBS supplemented with $10 \%(\mathrm{v} / \mathrm{v})$ DMSO and stored in liquid nitrogen until use.

\section{Statistical analysis}

Results for continuous variables are expressed as mean \pm SDs, and for dichotomous or categorical variables as percentages. Differences between groups were evaluated using Mann-Whitney $U$ test and Pearson's chi-square test, for continuous and categorical values, respectively. The association between TL and frailty or mortality was estimated by odds ratios (ORs) and their 95\% confidence intervals (CIs), obtained from logistic regression and the results were meta-analysed using a random effects method [27]. Variation/increase in $1 \mathbf{k b}$ was used for OR calculations. We built five logistic models. The first one (M1) without any adjustment. The second model (M2) adjusted for age (years) and sex. The third model (M3) additionally adjusted for diabetes mellitus (DM), cardiovascular disease (CVD) and cerebrovascular disease (CeVD). A fourth model (M4) further adjusted for education level (more than primary school), and, finally, the fifth model (M5) further adjusted for disability. $\boldsymbol{p}$ values $<0.05$ were considered statistically significant. Analyses were performed with the Statistical Packages Matrix and Metafor (version 2.0-0) in R for Windows version 2.15.2 [27].

\section{Results}

Characteristics of the TSHA study participants

The baseline characteristics of the TSHA participants are presented in Table 1. Frail subjects were older, had lower education and more often suffered from CVD than non-frail individuals. As expected, frail subjects showed higher dependency for BADL than non-frail subjects (Table 1). The mean TL was $10.24 \pm 2.92 \mathrm{~kb}$ among TSHA older subjects included in the study. No significant differences were detected in TL between frail $(10.16 \pm 3.02 \mathrm{~kb})$ and non-frail subjects $(10.26 \pm$ $2.89 \mathrm{~kb}$ ) (Table 1).

\section{Characteristics of the ENRICA study participants}

Frail subjects were older, more often female, and with more frequent hypertension and diabetes than the nonfrail group. Like for the TSHA participants, in the ENRICA cohort, the dependency for BADL was higher within the frail group. The mean TL was $10.02 \pm$ $2.91 \mathrm{~kb}$. No significant differences in TL were observed between frail and non-frail participants $(10.40 \pm 2.88 \mathrm{~kb}$ vs $9.93 \pm 2.92 \mathrm{~kb}$ ) (Table 2).

Telomere length is not associated with prevalent frailty in older subjects

In older subjects participating in TSHA, no association between TL and prevalent frailty was observed in the non-adjusted model (M1; OR: 0.99 [0.911.07], $p=0.78$ ). This lack of association was maintained after adjustment for age and sex (M2; OR: 1.05 [0.95-1.16], $p=0.34)$. Similar results were obtained after further adjustment for DM, cardio- and cerebrovascular disease (M3; OR: 1.06 [0.96-1.18], $p=0.23$ ), and by education level (M4; OR: 1.06 [0.96-1.18], $p=0.23)$. The same trend of this 
Table 1 Baseline characteristics of subjects in the Toledo Study for Healthy Aging (TSHA) cohort

\begin{tabular}{|c|c|c|c|c|}
\hline & All & Non-frail & Frail & $p$ value \\
\hline$N$ & 439 & 355 & 84 & \\
\hline Age & $75.9 \pm 6.6$ & $74.3 \pm 5.7$ & $82.7 \pm 5.4$ & $<0.001$ \\
\hline Male gender & $189(43.05)$ & $159(44.79)$ & $30(35.71)$ & 0.165 \\
\hline Hypertension & $282(64.53)$ & $222(62.54)$ & $60(73.17)$ & 0.091 \\
\hline Diabetes & $102(23.23)$ & $78(21.97)$ & $24(28.57)$ & 0.252 \\
\hline CVD & $51(11.62)$ & $34(9.58)$ & $17(20.24)$ & 0.011 \\
\hline $\mathrm{CeVD}$ & $20(4.57)$ & $15(4.24)$ & $5(5.95)$ & 0.699 \\
\hline Education level higher than basic school & $64(14.58)$ & $58(16.34)$ & $6(7.14)$ & 0.047 \\
\hline \multicolumn{5}{|l|}{ Frailty criteria $(\mathrm{FP})$} \\
\hline Low physical activity & $112(25.51)$ & $33(9.30)$ & $79(94.05)$ & $<0.001$ \\
\hline Weakness & $90(20.50)$ & $30(8.45)$ & $60(71.43)$ & $<0.001$ \\
\hline Weight loss & $41(9.47)$ & $25(7.08)$ & $16(20.00)$ & $<0.001$ \\
\hline Slowness & $59(15.13)$ & $22(6.41)$ & $37(78.72)$ & $<0.001$ \\
\hline Exhaustion & $113(25.80)$ & $41(11.55)$ & $72(86.75)$ & $<0.001$ \\
\hline Disability & $64(14.65)$ & $11(3.12)$ & $53(63.10)$ & $<0.001$ \\
\hline Telomere length $(\mathrm{kb})$ & $10.24 \pm 2.92$ & $10.26 \pm 2.89$ & $10.16 \pm 3.02$ & 0.813 \\
\hline
\end{tabular}

Data are expressed as mean $\pm \mathrm{SD}$ or as number $(\%)$. CeVD, cerebrovascular disease; $C V D$, cardiovascular disease; $F P$, frailty phenotype; $k b$, kilobases

association was maintained after additional adjustment for disability (M5; OR: 1.10 [0.97-1.23], $p=$ $0.13)$.
When we evaluated the association between TL and frailty in the ENRICA participants, similar results were obtained. In this sense, TL was not associated with

Table 2 Baseline characteristics of subjects in the ENRICA cohort

\begin{tabular}{|c|c|c|c|c|}
\hline & All & Non-frail & Frail & $p$ value \\
\hline$N$ & 430 & 355 & 75 & \\
\hline Age & $74.4 \pm 5.9$ & $73.8 \pm 5.6$ & $77.6 \pm 6.2$ & $<0.001$ \\
\hline Male gender & $178(41.40)$ & $156(43.94)$ & $22(29.33)$ & 0.027 \\
\hline Hypertension & $318(75.18)$ & $253(72.49)$ & $65(87.84)$ & 0.009 \\
\hline Diabetes & $108(25.23)$ & $76(21.53)$ & $32(42.67)$ & $<0.001$ \\
\hline CVD & $54(12.56)$ & $41(11.55)$ & $13(17.33)$ & 0.237 \\
\hline CeVD & $12(2.80)$ & $7(1.98)$ & $5(6.67)$ & 0.064 \\
\hline Education level higher than basic school & $195(45.35)$ & $173(48.73)$ & $22(29.33)$ & 0.003 \\
\hline \multicolumn{5}{|l|}{ Frailty criteria (FP) } \\
\hline Low physical activity & $90(21.03)$ & $44(12.46)$ & $46(61.33)$ & $<0.001$ \\
\hline Weakness & $160(37.38)$ & $96(27.20)$ & $64(85.33)$ & $<0.001$ \\
\hline Weight loss & 64 (14.99) & $26(7.37)$ & $38(51.35)$ & $<0.001$ \\
\hline Slowness & $66(15.46)$ & $27(6.41)$ & $39(52.70)$ & $<0.001$ \\
\hline Exhaustion & $79(18.37)$ & $23(6.48)$ & $56(74.67)$ & $<0.001$ \\
\hline Disability & $13(3.04)$ & $5(1.42)$ & $8(10.67)$ & $<0.001$ \\
\hline Telomere length (kb) & $10.02 \pm 2.91$ & $9.93 \pm 2.92$ & $10.40 \pm 2.88$ & 0.218 \\
\hline
\end{tabular}

Data are expressed as mean $\pm \mathrm{SD}$ or as number $(\%)$. $C e V D$, cerebrovascular disease; $C V D$, cardiovascular disease; $F P$, frailty phenotype; $k b$, kilobases 
prevalent frailty independently of the degree of adjustment: [(M1; OR: 1.06 [0.97-1.15], $p=0.21)$; (M2; OR: 1.06 [0.97-1.16], $p=0.19)$; (M3; OR: 1.06 [0.96-1.16], $p=0.25$ ); (M4; OR: 1.06 [0.96-1.16], $p=0.24)$; (M5, OR: 1.07 [0.98-1.18], $p=0.15)]$.

Meta-analysis of the results obtained from the two Spanish cohorts revealed that TL was not significantly associated with prevalent frailty (M1; OR: 1.02 [0.961.09 ], $p=0.54$ ). After adjustment for age and gender, the trend of the association was maintained (M2; OR: 1.06 [0.99-1.13], $p=0.11$ ). Same results were obtained after additional adjustments for diabetes, cardiovascular disease and cerebrovascular disease (M3; OR: 1.06 [0.99-1.13], $p=0.097$ ) and by education level (M4; OR: 1.06 [0.99-1.14], $p=0.093$ ). Further adjustment for disability (M5) did not materially change the results (OR: 1.08 [1.00-1.17], $p=0.04$ ).

Telomere length does not predict frailty

Among non-frailty participants at baseline, 10 (3.44\%) became frail at follow-up in the TSHA and 11 (5.09\%) in the ENRICA cohort. No association between TL and incident frailty risk was observed in either cohort in model M1 (Table 3). Results were not materially changed after further adjustment (Table 3). Accordingly, these null results also held after meta-analyses of data from both cohorts (Fig. 1a).

Table 3 Association between telomere length and the risk of frailty in TSHA and ENRICA participants

\begin{tabular}{|c|c|c|c|c|}
\hline & \multicolumn{2}{|l|}{ TSHA cohort } & \multicolumn{2}{|l|}{ ENRICA cohort } \\
\hline & OR $[95 \% \mathrm{CI}]$ & $p$ value & OR $[95 \% \mathrm{CI}]$ & $p$ value \\
\hline Model 1 & $0.88[0.71-1.10]$ & 0.27 & $1.07[0.88-1.31]$ & 0.49 \\
\hline Model 2 & $0.96[0.75-1.23]$ & 0.75 & $1.07[0.88-1.31]$ & 0.49 \\
\hline Model 3 & $0.97[0.75-1.27]$ & 0.84 & $1.08[0.88-1.31]$ & 0.45 \\
\hline Model 4 & $0.96[0.74-1.25]$ & 0.78 & 1.09 [0.89-1.34] & 0.42 \\
\hline Model 5 & $0.96[0.73-1.24]$ & 0.74 & 1.09 [0.89-1.34] & 0.42 \\
\hline
\end{tabular}

The association between telomere length and the risk for incident frailty was estimated by odds ratios (OR) and their $95 \%$ confidence intervals (CI, in brackets). Increase in TL by 1 kilobase was used for calculating OR. Model 1: non-adjusted model. Model 2: adjusted by age (years) and sex. Model 3: additionally adjusted by diabetes mellitus, cardiovascular disease, and cerebrovascular disease. Model 4: further adjusted by education level (higher than primary school). Model 5: further adjusted by disability in basic activities of daily living a

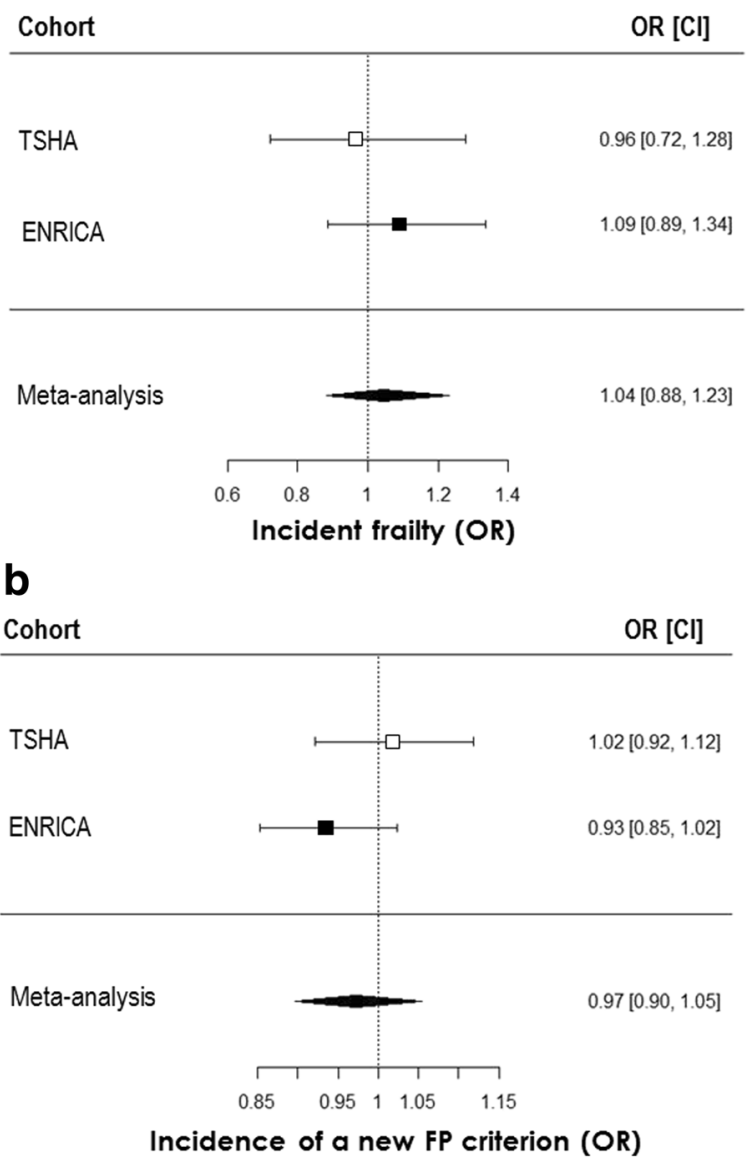

Fig. 1 Telomere length does not predict incident frailty and is not associated with the incidence of new frailty phenotype criteria in older subjects. Logistic regression of telomere length at baseline as independent variable and incident frailty defined by frailty phenotype along 3.5 years follow-up in subjects not frail at baseline (a) and logistic regression of telomere length at baseline as independent variable and incidence of acquisition of a new criterion of frailty phenotype along 3.5 years follow-up (b). Results from TSHA and ENRICA cohorts in separate and from the metaanalysis after fully adjustment model application (M5) are shown. Data are expressed as odds ratios (ORs) and 95\% confidence intervals (CIs). For panel a, $n$ was 291 (10 positive events) for TSHA and 216 (11 positive events) for ENRICA cohorts. $p>0.05$ for all associations. For panel b, $n$ was 303 ( 77 positive events) for TSHA and 256 (102 positive events) for ENRICA cohorts. $p>0.05$ for all associations

In addition, 46 out of 303 individuals in the TSHA cohort and 102 out of 256 in ENRICA study developed at least one new criterion of FP during follow-up. Again, TL did not show any association, either in any of the two cohorts or in the metaanalysis (Table 4, Fig. 1b). In the same regard, when we analysed the longitudinal relationship between 
Table 4 Association between telomere length and the risk for incidence of at least one new frailty phenotype criterion in TSHA and ENRICA participants

\begin{tabular}{|c|c|c|c|c|}
\hline & \multicolumn{2}{|l|}{ TSHA cohort } & \multicolumn{2}{|l|}{ ENRICA cohort } \\
\hline & OR $[95 \% \mathrm{CI}]$ & $p$ value & OR $[95 \% \mathrm{CI}]$ & $p$ value \\
\hline Model 1 & 0.99 [0.90-1.08] & 0.76 & $0.96[0.88-1.04]$ & 0.29 \\
\hline Model 2 & $1.01[0.92-1.11]$ & 0.89 & $0.95[0.87-1.04]$ & 0.25 \\
\hline Model 3 & $1.01[0.92-1.12]$ & 0.76 & $0.94[0.86-1.03]$ & 0.16 \\
\hline Model 4 & $1.02[0.92-1.12]$ & 0.75 & $0.94[0.86-1.03]$ & 0.16 \\
\hline Model 5 & $1.02[0.92-1.12]$ & 0.75 & $0.93[0.85-1.02]$ & 0.14 \\
\hline
\end{tabular}

The association between telomere length and the risk for incidence of a new frailty phenotype criterion was estimated by odds ratios (OR) and their $95 \%$ confidence intervals (CI, in brackets). Increase in TL by 1 kilobase was used for calculating OR. Model 1: nonadjusted model. Model 2: adjusted by age (years) and sex. Model 3: additionally adjusted by diabetes mellitus, cardiovascular disease and cerebrovascular disease. Model 4: further adjusted by education level (higher than primary school). Model 5: further adjusted by disability in basic activities of daily living

TL and each one of the FP criteria, no significant associations were detected (Fig. 2).

Telomere length does not predict mortality

During the same 3.5 years follow-up period, 46 out of 439 participants (10.48\%) from the TSHA cohort and 14 out of $430(4.13 \%)$ from the ENRICA cohort died, respectively. A higher death risk was observed within the frail group when compared to non-frail group: $30.95 \%$ vs. $5.63 \%, p<0.001$ for TSHA, and $9.43 \%$ vs. $3.15 \%, p=$ 0.08 for ENRICA. Moreover, the results of the metaanalysis showed a positive association between frailty status and the risk of death (OR: 5.59 [2.52-12.41], $p<0.001$ ), which was maintained after further adjustment for age and sex (OR: 4.08 [1.97-8.43], $p<0.001$ ).

By contrast, TL was not associated with mortality at 3.5 years follow-up in any of the cohorts in any of the analytical models (Table 5). When the results were meta-analysed, similar observations were obtained related to the lack of association between TL and mortality risk in older Spanish participants in this study. This was the case for the non-adjusted model (M1: OR: 0.97 [0.89-1.07], $p=0.59)$. Furthermore, there was no significant effect when adjusted by age and sex (M2: OR: 1.01 [0.90-1.14], $p=0.84$ ). This lack of association was also observed after several adjustments, including the fully adjustment model (M5) as it is shown in Fig. 3.

\section{Discussion}

Results from this study show that TL at baseline does not predict incident frailty nor mortality among community-dwelling older adults aged 65 years and older after a mean follow-up time of 3.5 years. This lack of association was observed regardless of the degree of adjustment for potential confounders, including age, sex, age-related diseases, education level or disability.

TL is widely accepted as a biomarker of ageing [8]. Although telomere shortening rate has been associated with reduced longevity and with increased incidence of age-related diseases in humans [28, 29], the relationship between telomere length and mortality is controversial, depending upon factors like ethnicity, gender and type of diseases $[9,30,31]$. On the other hand, frailty is a multidimensional and multifactorial syndrome associated with decreased functional capacity and increased vulnerability to minor external stressors and with increased risk of adverse outcomes [5]. It has been proposed that frailty is a biomarker of biological ageing [8], which leads us to expect a possible link between frailty status and TL. In fact, shorter TL was associated with decreased grip strength, one of the components of frailty syndrome in Chinese older persons [32]. In addition, although previous studies observed an association between TL and functional disability [33] and with one of the substrates of frailty, sarcopenia [34], conflicting results on the association between TL and frailty have been reported [6].

In this sense, the findings of the cross-sectional analysis carried out in TSHA and in ENRICA cohorts in addition to the results of the meta-analysis revealed no relation between TL and prevalent frailty in older population. In fact, no significant association was observed between TL and frailty assessed at baseline by Fried's criteria in nonadjusted model, corroborating the findings of other authors $[11,12,14,35,36]$. Although one study has found a crosssectional association between TL and frailty, this study shows strong differences with ours in terms of ethnicity, higher proportion of female subjects (65.6\%), the inclusion of younger subjects (more than 50\% are 60-69 years old) or the higher prevalence of frailty $(25 \%)$ [13].

After a final adjustment for disability, a marginal association between TL and frailty was observed in the meta-analysis, showing a higher risk for frailty when TL increased. This unexpected marginal positive association could be related to the existence of a survival bias when studying older subjects, as it has been reported in a recent meta-analysis [37]. 
a

b

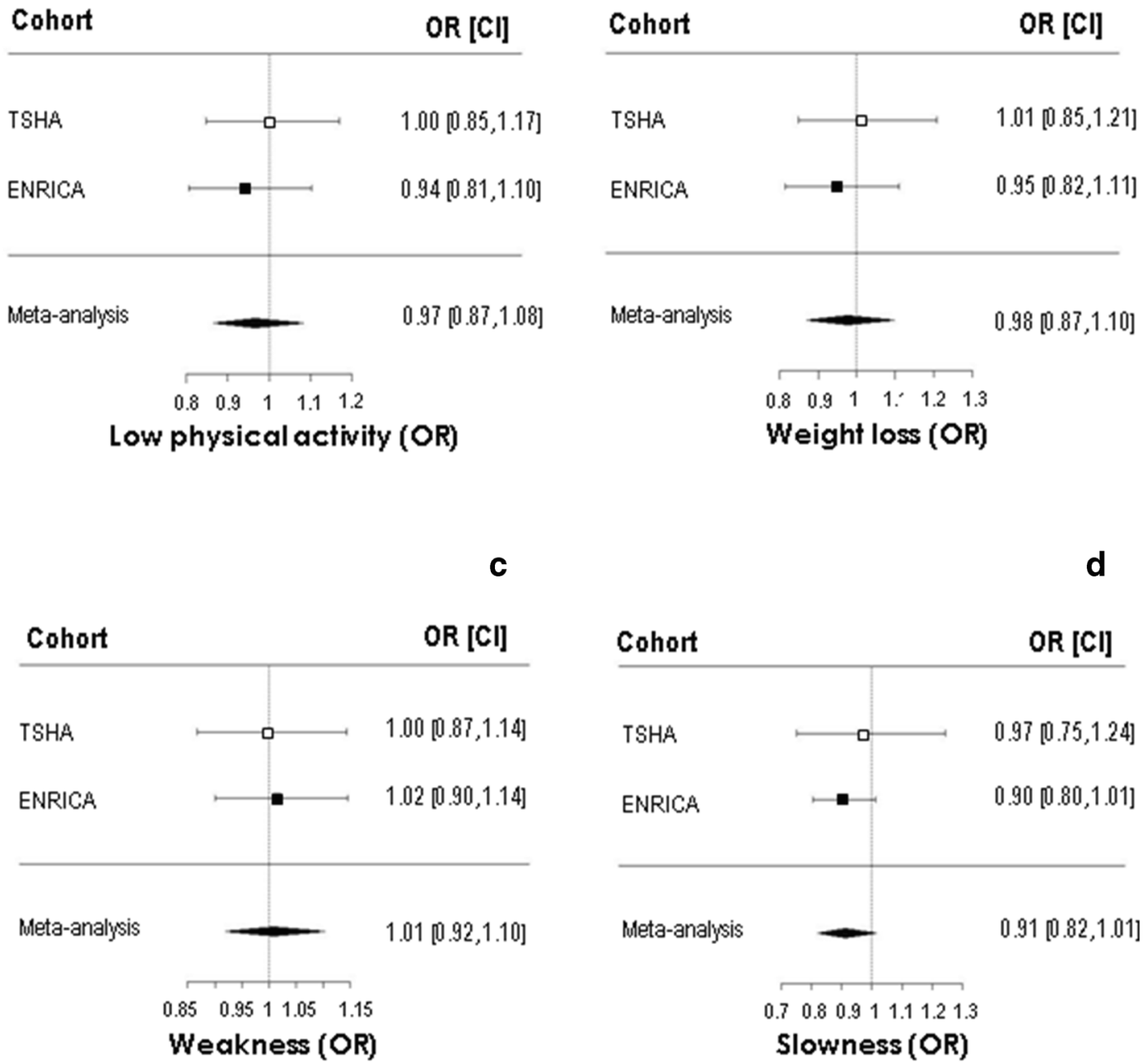

e

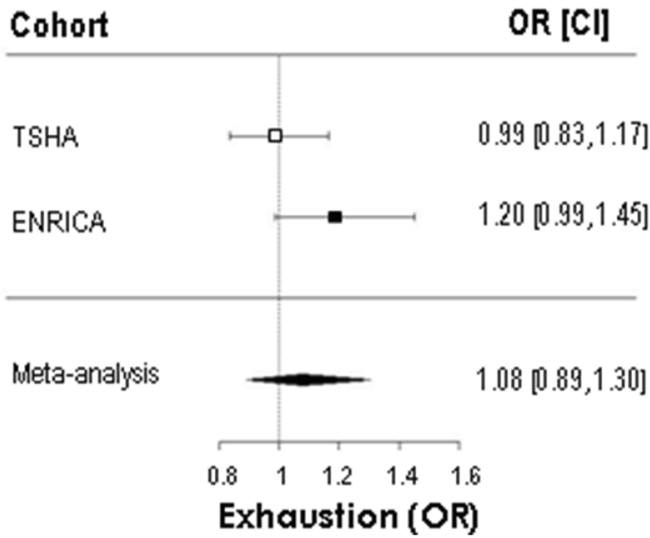


Fig. 2 Telomere length is not associated with the incidence of specific new frailty phenotype criteria. Logistic regression of telomere length at baseline as independent variable and incidence of acquisition of low physical activity (a), weight loss (b), weakness (c), slowness (d), and exhaustion (e) along 3.5 years follow-up. Results from TSHA and ENRICA cohorts in separate and from the meta-analysis after fully adjustment model application (M5) are shown. Data are expressed as odds ratios (ORs) and $95 \%$ confidence intervals (CIs). $p>0.05$ for all associations

The assumption of the lack of association between TL and frailty raising from cross-sectional analysis is reinforced by the absence of relationships between physical performance and TL shortening, by measuring TL in two time points. Telomere shortening has not shown to be cross-sectionally associated with frailty or other physical performance determinations $[38,39]$.

Despite the fact that cross-sectional studies analysing the relationship between TL and frailty prevalence are relatively abundant, longitudinal analysis of the influence of TL on incident frailty are scarce. Our longitudinal data suggest that TL at baseline does not influence the risk of incident frailty after 3.5 years follow-up. It should be mentioned that one previous study addressed the relationship between TL and incident frailty as a side outcome at 4 years follow-up in older Chinese people. The study found a similar lack of association between TL and incident frailty [11]. On the other hand, analysis of incident frailty by $\mathrm{Yu}$ and collaborators [11] was somewhat different. They compared robust participants

Table 5 Association between TL and mortality in TSHA and ENRICA participants

\begin{tabular}{|c|c|c|c|c|}
\hline & \multicolumn{2}{|l|}{ TSHA cohort } & \multicolumn{2}{|l|}{ ENRICA cohort } \\
\hline & OR $[95 \% \mathrm{CI}]$ & $p$ value & OR $[95 \% \mathrm{CI}]$ & $p$ value \\
\hline Model 1 & $1.00[0.90-1.11]$ & 1.00 & $0.89[0.74-1.08]$ & 0.25 \\
\hline Model 2 & 1.05 [0.94-1.18] & 0.39 & $0.92[0.76-1.13]$ & 0.43 \\
\hline Model 3 & $1.08[0.96-1.21]$ & 0.22 & $0.94[0.77-1.16]$ & 0.58 \\
\hline Model 4 & 1.08 [0.96-1.21] & 0.20 & $0.93[0.76-1.15]$ & 0.52 \\
\hline Model 5 & $1.08[0.96-1.22]$ & 0.21 & $0.95[0.77-1.18]$ & 0.66 \\
\hline
\end{tabular}

The association between telomere length and mortality was estimated by odds ratios (OR) and their $95 \%$ confidence intervals (CI, in brackets). Increase in TL by 1 kilobase was used for calculating OR. Model 1: non-adjusted model. Model 2: adjusted by age (years) and sex. Model 3: additionally adjusted by diabetes mellitus, cardiovascular disease and cerebrovascular disease. Model 4: further adjusted by education level (higher than primary school). Model 5: further adjusted by disability in basic activities of daily living that remained robust vs those who became non-robust (i.e., pre-frail and frail) along the follow-up, while we have assessed those non-frail (i.e., robust plus pre-frail) who became frail vs those remaining in the basal status, which, in our opinion, is a more accurate way to determine incident frailty. Moreover, it is of importance to confirm these evidences in populations with different ethnic backgrounds since differences in TL shortness by race/ethnicity have been previously proposed [40] and even the characteristics of the relationship between TL and frailty also seem to be ethnicity dependent $[31,41]$.

Furthermore, the lack of influence of TL on future physical performance was supported in our study by the lack of association of TL at baseline with the development of a new FP criterion in our older population during follow-up. This analysis allows for increasing the numbers of positive events, suggesting that a low number of events is not responsible for the lack of association between TL and incident frailty. Finally, the concept is further reinforced with the lack of association of TL with the acquisition of any of the specific FP criteria. A previous longitudinal study in a European population including older subjects from the Netherlands Study of Depression in Older persons, with a 2year follow-up period, although did not specifically address the influence of baseline TL on incident frailty, evidenced that TL was not significantly associated with changes in frailty parameters (Fried's criteria) during the studied period [42]. Therefore, the results of our crosssectional and longitudinal analysis support previous suggestions made by other authors proposing that TL is not a meaningful biomarker of frailty $[6,41]$.

Moreover, we assessed the influence of TL on mortality risk and evidenced that this parameter also failed to be predictive of mortality in this aged population independently of the model used for adjustment. Although short telomeres have been associated with reduced longevity and several studies have shown that short telomeres are associated with increased all-cause mortality risk in the general population [37], the magnitude of this association seems to decrease with increasing age [43]. In this sense, previous studies showed inverse association between TL and mortality in subjects aged 60 and 75 years [9] but not in subjects aged over 80 years old [44]. More recent reports have also failed to detect any association between TL and mortality in older populations [30].

Furthermore, frailty seems to be a better predictor of mortality than TL since our results revealed a 
Fig. 3 Telomere length does not predict mortality in older subjects. Logistic regression of telomere length at baseline as independent variable and death along 3.5 years follow-up. Results from TSHA and ENRICA cohorts in separate and from the meta-analysis after fully adjustment model application (M5) are shown. Data are expressed as odds ratios (ORs) and $95 \%$ confidence intervals (CIs). $n$ was 439 (46 deaths) for TSHA and 430 (14 deaths) for ENRICA cohorts. $p>0.05$ for all associations

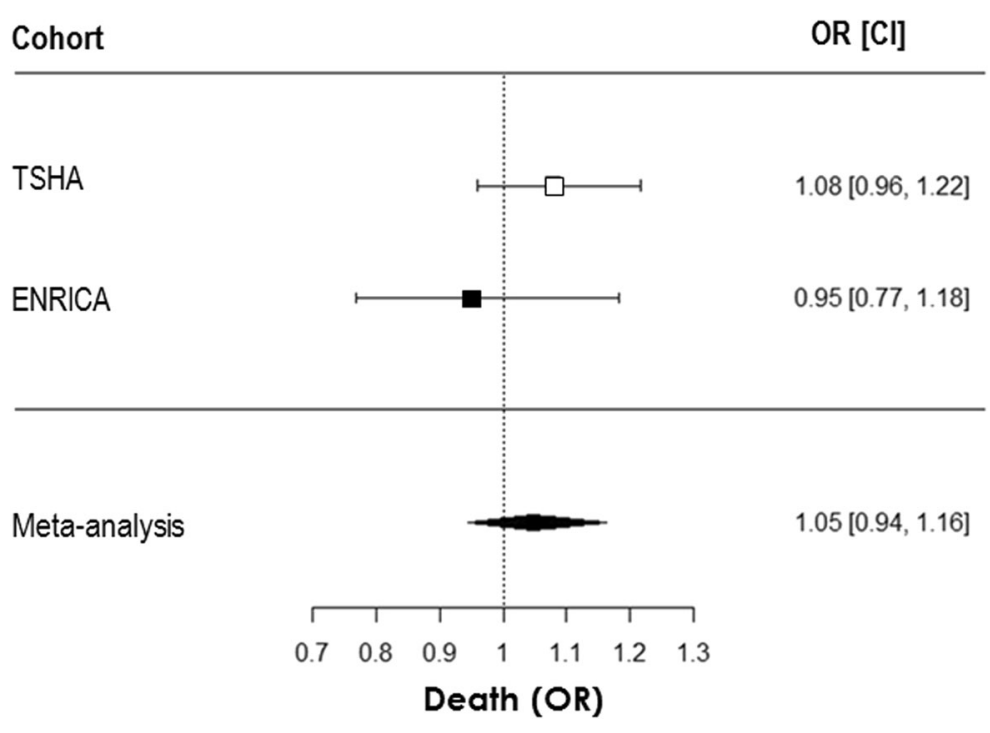

is not especially worthy, assessed in terms of both physical function and longevity. Accordingly, the role of telomere length would be relevant in earlier stages of life. Frailty is, in any case, one step in the ageing process, preceding disability. What we show in our study is that telomere length is not able to discriminate the frailty status nor to predict it, suggesting that telomere length is not a relevant issue among the biological factors leading or underlying frailty, like it is the case for inflammation, vascular function or insulin resistance [5]. This would be in agreement with the concept that ageing has no distinct genetic signature and is the result of simultaneous damage accumulation processes [47]. This means that no singular process is behind all manifestations of ageing, hampering the possibility of finding a single biomarker of biological ageing [48].

In conclusion, the analysis of two well-characterized cohorts of Spanish community-dwelling older adults shows that TL does not predict incident frailty or mortality. This suggests that TL is not a reliable biomarker of functional age.

Acknowledgments We appreciate the altruistic contribution of the participants from the Toledo Study for Healthy Aging cohort and ENRICA cohort.

Authors' contributions MEA, JA, JAC, SW, FRA and LRM participated in conceptualization and design of the study; MEA, JA, JAC, SW, JGM, IGP, FLGG, FRA and LRM performed data acquisition analysis and interpretation; MEA, JA, JAC, SW, FRA and LRM wrote the original draft of the manuscript; all authors reviewed, edited and approved the final version of the manuscript. 
Funding This work has received funding from the European Union's Seventh Framework Programme (FP7/2007-2013) under grant agreement number 305483 and was supported by grants from the Ministry of Economy and Competitiveness and cofinanced by FEDER funds (Instituto de Salud Carlos III, PI15/00674, PI15/01160) and CIBERFES (CB16/10/00464), Spanish Government.Data availabilityNot applicable.

\section{Compliance with ethical standards}

Conflict of interest The authors declare that they have no conflict of interest.

Ethics approval The study protocols were approved by the Clinical Research Ethics Committee of the Complejo Hospitalario de Toledo (Toledo Study for Healthy Aging cohort), and by the Clinical Research Ethics Committee of the 'La Paz' University Hospital in Madrid (ENRICA cohort).

Consent to participate All participants gave written informed consent to participate.

Code availability Not applicable.

\section{References}

1. United Nations. World population ageing (2019). https://www.un.org/en/development/desa/population /publications/pdf/ageing/WorldPopulationAgeing2019Highlights.pdf.

2. Dzau VJ, Inouye SK, Rowe JW, Finkelman E, Yamada T. Enabling healthful aging for all - the National Academy of Medicine Grand Challenge in Healthy Longevity. N Engl J Med. 2019;381:1699-701. https://doi.org/10.1056 /NEJMp1912298.

3. Brivio P, Paladini MS, Racagni G, Riva MA, Calabrese F, Molteni R. From healthy aging to frailty: in search of the underlying mechanisms. Curr Med Chem. 2019;26:3685701. https://doi.org/10.2174/0929867326666190717 152739 .

4. Dent E, Martin FC, Bergman H, Woo J, Romero-Ortuño R, Walston JD. Management of frailty: opportunities, challenges, and future directions. Lancet. 2019;394:1376-86. https://doi.org/10.1016/S0140-6736(19)31785-4.

5. Angulo J, El Assar M, Rodriguez-Manas L. Frailty and sarcopenia as the basis for the phenotypic manifestation of chronic diseases in older adults. Mol Asp Med. 2016;50:132. https://doi.org/10.1016/j.mam.2016.06.001.

6. Lorenzi M, Bonassi S, Lorenzi T, Giovannini S, Bernabei R, Onder G. A review of telomere length in sarcopenia and frailty. Biogerontology. 2018;19:209-21.

7. Ferrucci L, Gonzalez-Freire M, Fabbri E, Simonsick E, Tanaka T, Moore Z, et al. Measuring biological aging in humans: a quest. Aging Cell. 2020;19:e13080. https://doi. org/10.1111/acel.13080.
8. Fasching CL. Telomere length measurement as a clinical biomarker of aging and disease. Crit Rev Clin Lab Sci. 2018;55:443-65. https://doi.org/10.1080/104083 63.2018 .1504274 .

9. Cawthon RM, Smith KR, O'Brien E, Sivatchenko A, Kerber RA. Association between telomere length in blood and mortality in people aged 60 years or older. Lancet. 2003;361:393-5. https://doi.org/10.1016/S0140-6736(03 )12384-7.

10. El Assar M, Angulo J, Rodríguez-Mañas L. Frailty as a phenotypic manifestation of underlying oxidative stress. Free Radic Biol Med. 2020;149:72-7. https://doi. org/10.1016/j.freeradbiomed.2019.08.011.

11. Yu R, Tang N, Leung J, Woo J. Telomere length is not associated with frailty in older Chinese elderly: crosssectional and longitudinal analysis. Mech Ageing Dev. 2015;152:74-9. https://doi.org/10.1016/j.mad.2015.10.002.

12. Breitling LP, Saum KU, Perna L, Schottker B, Holleczek B, Brenner $\mathrm{H}$. Frailty is associated with the epigenetic clock but not with telomere length in a German cohort. Clin Epigenetics. 2016;8:21. https://doi.org/10.1186/s13148016-0186-5.

13. Ortiz-Ramírez M, Sánchez-García S, García-De la Torre P, Reyes-Maldonado E, Sánchez-Arenas R, Rosas-Vargas H. Telomere shortening and frailty in Mexican older adults. Geriatr Gerontol Int. 2018;18:1286-92. https://doi. org/10.1111/ggi.13463.

14. Vetter VM, Spira D, Banszerus VL, Demuth I. Epigenetic clock and leukocyte telomere length are associated with vitamin $\mathrm{D}$ status, but not with functional assessments and frailty in the Berlin Aging Study II. J Gerontol A Biol Sci Med Sci. 2020; (On-line ahead of print). https://doi. org/10.1093/gerona/glaa101.

15. García-García FJ, Gutiérrez Avila G, Alfaro-Acha A, et al. The prevalence of frailty syndrome in an older population from Spain. The Toledo Study for Healthy Aging. J Nutr Health Aging. 2011;15:852-6. https://doi.org/10.1007 /s12603-011-0075-8.

16. Sánchez-Sánchez JL, Izquierdo M, Carnicero-Carreño JA, García-García FJ, Rodríguez-Mañas L. Physical activity trajectories, mortality, hospitalization, and disability in the Toledo Study of Healthy Aging. J Cachexia Sarcopenia Muscle. 2020; (On-line ahead of print). https://doi. org/10.1002/jcsm. 12566 .

17. Rodríguez-Artalejo F, Graciani A, Guallar-Castillón P, León-Muñoz LM, Zuluaga MC, López-García E, et al. Rationale and methods of the study on nutrition and cardiovascular risk in Spain (ENRICA). Rev Esp Cardiol. 2011;64:876-82. https://doi.org/10.1016/j. recesp.2011.05.019.

18. Ortolá R, Struijk EA, García-Esquinas E, RodríguezArtalejo F, López-García E. Changes in dietary intake of animal and vegetable protein and unhealthy aging. Am J Med. 2019;132:1091-102. e9. https://doi.org/10.1016/j. amjmed.2019.06.051.

19. Fried LP, Tangen CM, Walston J, Newman AB, Hirsch C, Gottdiener J, et al. Frailty in older adults: evidence for a phenotype. J Gerontol A Biol Sci Med Sci. 2001;56:M14656. https://doi.org/10.1093/gerona/56.3.m146.

20. Ottenbacher KJ, Branch LG, Ray L, Gonzales VA, Peek MK, Hinman MR. The reliability of upper- and lower- 
extremity strength testing in a community survey of older adults. Arch Phys Med Rehabil. 2002;83:1423-7. https://doi.org/10.1053/apmr.2002.34619.

21. Guralnik JM, Simonsick EM, Ferrucci L, Glynn RJ, Berkman LF, Blazer DG, et al. A short physical performance battery assessing lower extremity function: association with self-reported disability and prediction of mortality and nursing home admission. J Gerontol. 1994;49:M85-94. https://doi.org/10.1093/geronj/49.2.m85.

22. Washburn RA, Smith KW, Jette AM, Janney CA. The physical activity scale for the elderly (PASE): development and evaluation. J Clin Epidemiol. 1993;46:153-62. https://doi.org/10.1016/0895-4356(93)90053-4.

23. Katz S, Ford AB, Moskowitz RW, Jackson BA, Jaffe MW. Studies of illness in the aged. The index of ADL: a standardized measure of biological and psychosocial function. JAMA. 1963;185:914-9. https://doi.org/10.1001 /jama.1963.03060120024016.

24. de Pedro N, Díez M, García I, García J, Otero L, Fernández L, et al. Analytical Validation of Telomere Analysis Technology ${ }^{\circledR}$ for the high-throughput analysis of multiple telomere-associated variables. Biol Proced Online. 2020;22: 2. https://doi.org/10.1186/s12575-019-0115-z.

25. Cawthon RM. Telomere measurement by quantitative PCR. Nucleic Acids Res. 2002;30:e47. https://doi.org/10.1093 /nar/30.10.e47.

26. O'Callaghan NJ, Fenech M. A quantitative PCR method for measuring absolute telomere length. Biol Proced Online. 2011;13:3. https://doi.org/10.1186/1480-9222-13-3.

27. Viechtbauer W. Conducting meta-analyses in $\mathrm{R}$ with the metafor package. J Stat Softw. 2010;36:1-48. https://doi. org/10.18637/jss.v036.i03.

28. Muezzinler A, Zaineddin AK, Brenner H. A systematic review of leukocyte telomere length and age in adults. Ageing Res Rev. 2013;12:509-19. https://doi.org/10.1016 /j.arr.2013.01.003.

29. Blackburn EH, Epel ES, Lin J. Human telomere biology: a contributory and interactive factor in aging, disease risk, and protection. Science. 2015;350:1193-8. https://doi. org/10.1126/science.aab3389.

30. Svensson J, Karlsson MK, Ljunggren O, Tivesten A, Mellstrom D, Moverare-Skrtic S. Leukocyte telomere length is not associated with mortality in older men. Exp Gerontol. 2014;57:6-12. https://doi.org/10.1016/j.exger.2014.04.013.

31. Needham BL, Rehkopf D, Adler N, Gregorich S, Lin J, Blackburn EH, et al. Leukocyte telomere length and mortality in the National Health and Nutrition Examination Survey, 1999-2002. Epidemiology. 2015;26:528-35. https://doi. org/10.1097/EDE.0000000000000299.

32. Woo J, Yu R, Tang N, Leung J. Telomere length is associated with decline in grip strength in older persons aged 65 years and over. Age (Dordr). 2014;36:9711. https://doi. org/10.1007/s11357-014-9711-7.

33. Risques RA, Arbeev KG, Yashin AI, Ukraintseva SV, Martin GM, Rabinovitch PS, et al. Leukocyte telomere length is associated with disability in older U.S. population.
J Am Geriatr Soc. 2010;58:1289-98. https://doi. org/10.1111/j.1532-5415.2010.02948.x.

34. Marzetti E, Lorenzi M, Antocicco M, Bonassi S, Celi M, Mastropaolo S, et al. Shorter telomeres in peripheral blood mononuclear cells from older persons with sarcopenia: results from an exploratory study. Front Aging Neurosci. 2014;6:233. https://doi.org/10.3389/fnagi.2014.00233.

35. Saum KU, Dieffenbach AK, Muezzinler A, et al. Frailty and telomere length: cross-sectional analysis in 3537 older adults from the ESTHER cohort. Exp Gerontol. 2014;58:250-5. https://doi.org/10.1016/j.exger.2014.08.009.

36. Zhou J, Wang J, Shen Y, Yang Y, Huang P, Chen S, et al. The association between telomere length and frailty: a systematic review and meta-analysis. Exp Gerontol. 2018;106: 16-20. https://doi.org/10.1016/j.exger.2018.02.030.

37. Wang Q, Zhan Y, Pedersen NL, Fang F, Hagg S. Telomere length and all-cause mortality: a meta-analysis. Ageing Res Rev. 2018;48:11-20. https://doi.org/10.1016/j. arr.2018.09.002.

38. Gardner MP, Martin-Ruiz C, Cooper R, Hardy R, Sayer AA, Cooper $\mathrm{C}$, et al. Telomere length and physical performance at older ages: an individual participant meta-analysis. PLoS One. 2013;8:e69526. https://doi.org/10.1371/journal. pone.0069526.

39. Haapanen MJ, Perala MM, Salonen MK, et al. Telomere length and frailty: the Helsinki Birth Cohort study. J Am Med Dir Assoc. 2018;19:658-62. https://doi.org/10.1016/j. jamda.2018.05.011.

40. Diez Roux AV, Ranjit N, Jenny NS, Shea S, Cushman M, Fitzpatrick A, et al. Race/ethnicity and telomere length in the Multi-Ethnic Study of Atherosclerosis. Aging Cell. 2009;8: 251-7. https://doi.org/10.1111/j.1474-9726.2009.00470.x.

41. Araujo Carvalho AC, Tavares Mendes ML, da Silva Reis MC, Santos VS, Tanajura DM, Martins-Filho PRS. Telomere length and frailty in older adults-a systematic review and meta-analysis. Ageing Res Rev. 2019;54: 100914. https://doi.org/10.1016/j.arr.2019.100914.

42. Arts MHL, Collard RM, Comijs HC, de Jonge L, Penninx BWJH, Naarding P, et al. Leucocyte telomere length is no molecular marker of physical frailty in late-life depression. Exp Gerontol. 2018;111:229-34. https://doi.org/10.1016/j. exger.2018.07.016.

43. Zhan Y, Liu XR, Reynolds CA, Pedersen NL, Hagg S, Clements MS. Leukocyte telomere length and all-cause mortality: a between-within twin study with timedependent effects using generalized survival models. Am J Epidemiol. 2018;187:2186-91. https://doi.org/10.1093 /aje/kwy128.

44. Martin-Ruiz CM, Gussekloo J, van Heemst D, von Zglinicki T, Westendorp RG. Telomere length in white blood cells is not associated with morbidity or mortality in the oldest old: a population-based study. Aging Cell. 2005;4:287-90. https://doi.org/10.1111/j.1474-9726.2005.00171.x.

45. Rodríguez-Mañas L, Féart C, Mann G, Viña J, Chatterji S, Chodzko-Zajko W, et al. Searching for an operational definition of frailty: a Delphi method based consensus statement: the frailty operative definition-consensus conference project. 
J Gerontol A Biol Sci Med Sci. 2013;68:62-7. https://doi. org/10.1093/gerona/gls119.

46. Fitzpatrick AL, Kronmal RA, Kimura M, Gardner JP, Psaty BM, Jenny NS, et al. Leukocyte telomere length and mortality in the Cardiovascular Health Study. J Gerontol A Biol Sci Med Sci. 2011;66:421-9. https://doi.org/10.1093 /gerona/glq224.

47. Gladyshev VN. Aging progressive decline in fitness due to the rising deleteriome adjusted by genetic, environmental, and stochastic processes. Aging Cell. 2016;15:594-602. https://doi.org/10.1111/acel.12480.
48. Galkin F, Mamoshina P, Aliper A, de Magalhaes JP, Gladyshev VN, Zhavoronkov A. Biohorology and biomarkers of aging: current state-of-the-art, challenges and opportunities. Ageing Res Rev. 2020;60:101050. https://doi.org/10.1016/j.arr.2020.101050.

Publisher's note Springer Nature remains neutral with regard to jurisdictional claims in published maps and institutional affiliations. 